\title{
Géolinguistique
}

17 | 2017

Varia

\section{Geolingüística de los lenguajes silbados del mundo, con un enfoque en el español silbado}

Géolinguistique des langues sifflées dans le monde, avec un accent particulier sur l'espagnol sifflé

Geolinguistics of the World's Whistled Languages, with a Focus on Whistled Spanish

Julien Meyer y David Díaz Reyes

\section{OpenEdition}

\section{Journals}

Edición electrónica

URL: http://journals.openedition.org/geolinguistique/373

DOI: 10.4000/geolinguistique.373

ISSN: $2650-8176$

\section{Editor}

UGA Éditions/Université Grenoble Alpes

\section{Edición impresa}

Fecha de publicación: 1 diciembre 2017

Paginación: 99-124

ISBN: 978-2-37747-025-9

ISSN: 0761-9081

\section{Referencia electrónica}

Julien Meyer y David Díaz Reyes, « Geolingüística de los lenguajes silbados del mundo, con un enfoque en el español silbado », Géolinguistique [En línea], 17 | 2017, Publicado el 01 febrero 2019 consultado el 29 octubre 2020. URL : http://journals.openedition.org/geolinguistique/373 ; DOI : https://doi.org/10.4000/geolinguistique.373 


\title{
Geolingüística de los lenguajes silbados del mundo, con un enfoque en el español silbado
}

\author{
Julien Meyer \\ Université Grenoble Alpes, GIPSA-lab UMR 5216 CNRS, Francia \\ Asociación de Investigación "El Mundo Silba», Paris, Francia \\ David Díaz Reyes \\ Asociación de Investigación «Yo Silbo», Canarias, España
}

\section{Resumen}

Este artículo aborda el tema de las variaciones geo-lingüísticas de una práctica tradicional especial y poco conocida de lenguaje basada en silbidos llamada «habla silbada» (o a veces, lenguaje silbado), y que consiste en hablar por medio de estos silbidos con el fin de comunicarse a largas distancias. Lo que se produce es una melodía que imita el habla modal y que resulta inteligible para los interlocutores. En esteartículo primero veremos la diversidad de idiomas que se silban en el planeta y cómo este fenómeno se adapta a la variación de las estructuras lingüísticas. En segundo lugar comprobaremos que las poblaciones del mundo que han desarrollado este tipo de forma silbada de su idioma viven en entornos naturales que favorecen el aislamiento de los individuos durante sus actividades rurales diarias. De hecho, los lenguajes silbados existen en contextos geolingüísticos particulares que comparten la combinación de la presencia de las actividades tradicionales de subsistencia (caza, pastoreo, agricultura de montaña) con ciertos tipos de hábitats (bosques y montañas). Por último, presentaremos las variaciones geo-lingüísticas que existen o han existido en un mismo idioma con el ejemplo del español silbado que se ha observado hasta hoy en varias de las Islas Canarias, pero también en México y en Andalucía. 


\title{
Palabras clave
}

Enguaje silbado, habla silbada, silbo, español, Islas, Canarias, tipología.

\section{Résumé}

Cet article aborde le thème des variations géolinguistiques d'une pratique langagière traditionnelle spéciale et peu connue basée sur le sifflement et appelée "parole sifflée» (ou parfois "langue sifflée »), qui consiste à parler en sifflant pour communiquer à grande distance. Le résultat est une mélodie qui imite la parole modale et qui reste intelligible pour les interlocuteurs. Dans cet article nous verrons premièrement la diversité des langues qui sont sifflées sur la planète et comment ce phénomène s'adapte à la variation des structures linguistiques. Dans un deuxième temps nous verrons que les populations du monde qui ont développé ce type de forme sifflée de leur langue vivent dans des environnements naturels qui favorisent l'isolement des individus lors de leurs activités rurales quotidiennes. On rencontre en effet la parole sifflée dans des contextes géolinguistiques particuliers qui ont en commun la combinaison de la présence d'activités traditionnelles de subsistance (chasse, pastoralisme, agriculture de montagne) avec certains types de biotopes (forêts et montagnes). Finalement, nous présenterons les variations géolinguistiques qui existent ou ont existé dans une même langue, avec l'exemple de l'espagnol sifflé qui a été observé jusqu'à aujourd'hui dans plusieurs des îles Canaries, mais aussi à Mexico et en Andalousie.

\section{Mots-clés}

Langue sifflée, parole sifflée, Silbo, variations linguistiques, îles Canaries, espagnol.

\begin{abstract}
This article deals with the theme of geolinguistic variations of a special and little known traditional language practice which is based on whistling and called "whistled speech" (or sometimes "whistled languages"), which consists in speaking while whistling to communicate at long distance. The result is a melody which imitates modal speech and that remains intelligible for the interlocutors. We will first explore the diversity of languages that are whistled around the globe and how this phenomenon adapts to the variation of structures of different languages. Next we will observe that the populations that have developed this type of whistled form of their language live in natural environments that favor the isolation of individuals during their daily rural activities. Whistled speech exists in particular geolinguistic contexts that have in common the combination of
\end{abstract}


the presence of traditional activities of subsistence (hunting, pastoralism, hill agriculture) with specific types of biotopes (dense forests and mountains). Finally, we present the geolinguistic variations that exist or have existed in a same language with the example of whistled Spanish which has been so far observed in several Canary Islands, but also in Mexico and in Andalusia.

\section{Keywords}

Whistled language, whistled speech, Silbo, linguistic variations, Canary Islands, Spanish.

\section{Introducción}

Este artículo trata sobre variaciones geolingüísticas en una práctica tradicional de lenguaje muy especial y poco conocida basada en silbidos llamada «lenguaje silbado» (o a veces «lengua silbada»), que consiste en hablar silbando para comunicarse a larga distancia. En realidad, técnicamente no puede hablarse de nuevos lenguajes ni de dialectos de la lengua nativa, sino más bien de una extensión de la misma. La única diferencia es que las palabras se articulan como silbidos, es decir, los sonidos no son generados por la vibración de las cuerdas vocales como en el habla con voz, sino por una corriente de aire comprimido en la boca que se moldea con la lengua, la mandíbula, los labios y los dedos (fig. 1). A veces, la fuente del sonido es producida con otros elementos como una hoja que vibra entre los labios (fig. 2) o con la resonancia del flujo de aire dentro de una cavidad formada en frente de la boca por las manos (fig. 3). El resultado es una melodía que imita las sílabas del habla ordinaria (el «habla ordinaria» esta también llamada «habla modal» por los especialistas de fonética) y que resulta inteligible por los interlocutores.

Muchas poblaciones del mundo que viven en entornos naturales que propician el aislamiento de individuos durante las actividades tradicionales de subsistencia (como el pastoreo o la caza) han desarrollado una forma silbada de su lengua para comunicarse a distancia. Silbar es uno de los múltiples modos de expresión para estos idiomas del mundo. Al final, la versión silbada de un idioma es un verdadero sistema de telecomunicación antiguo y un modo complementario de expresión de la forma hablada, al igual que el susurro, el grito o el canto.

A lo largo de este artículo veremos primero la diversidad de lenguas que son silbadas en el mundo, y cómo este fenómeno se adapta a la variación 
de estructuras lingüísticas. Hasta la fecha, en todo el mundo se conocen alrededor de 40 poblaciones remotas y de baja densidad que han desarrollado su idioma local en este registro del habla (fig. 4), y hay indicios de que muchas más han tenido, o todavía tienen, esta tradición (cerca de 70) (tabla 1). En una segunda parte veremos que esta práctica se encuentra en contextos geolingüísticos particulares, que tienen en común la combinación de la presencia de actividades tradicionales de subsistencia (caza, pastoreo, agricultura de monte) con ciertos tipos de entornos naturales o biotopos (bosques y montañas). Finalmente, ilustraremos la variación de prácticas del lenguaje silbado que existe o que ha existido en una misma lengua con el ejemplo del español silbado que ha sido observado hasta la fecha en varias islas del Archipiélago Canario, pero también en México y en Andalucía.

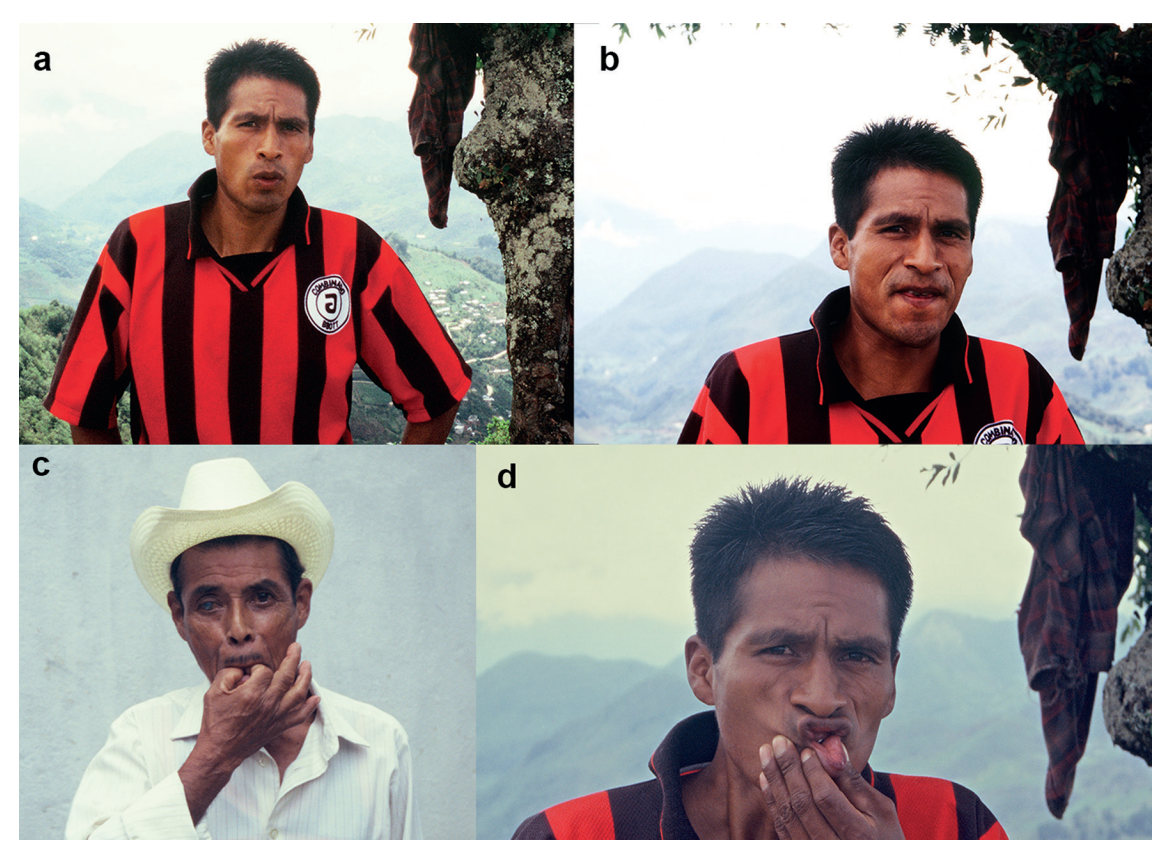

Figura 1. - Diferentes técnicas de silbar ilustradas aquí por dos silbadores mazatecos de México. a. Bulto bilabial; b. Lingo-dental; c. Con dedos insertos en la boca; $d$. Labio inferior (con un flujo de aire ingresivo en este caso). 


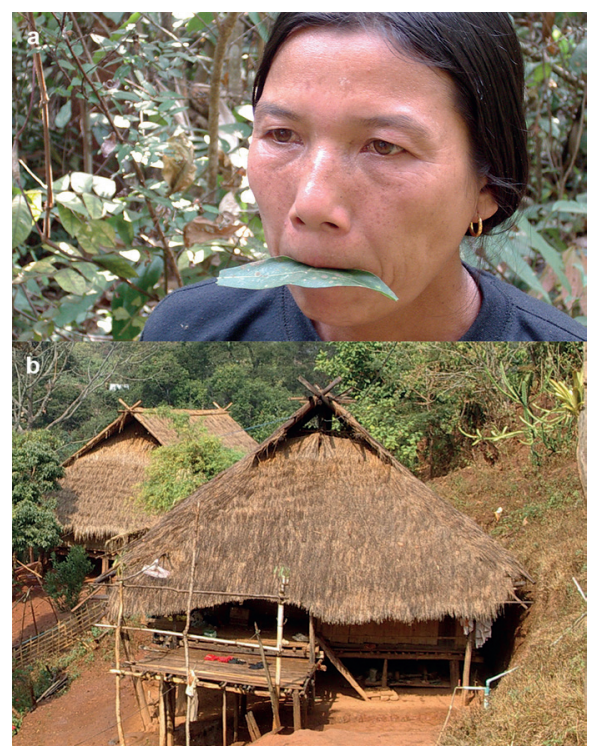

Figura 2. - a. Técnica con hoja ilustrada por una mujer de la población Akha del norte de Tailandia; $b$. Casa típica en un pueblo Akha, cerca de la ciudad de Chiang Rai.

Fotos: agradecimientos a Julien Meyer y Laure Dentel.

(C) Julien Meyer y Laure Dentel. Todos los derechos reservados.

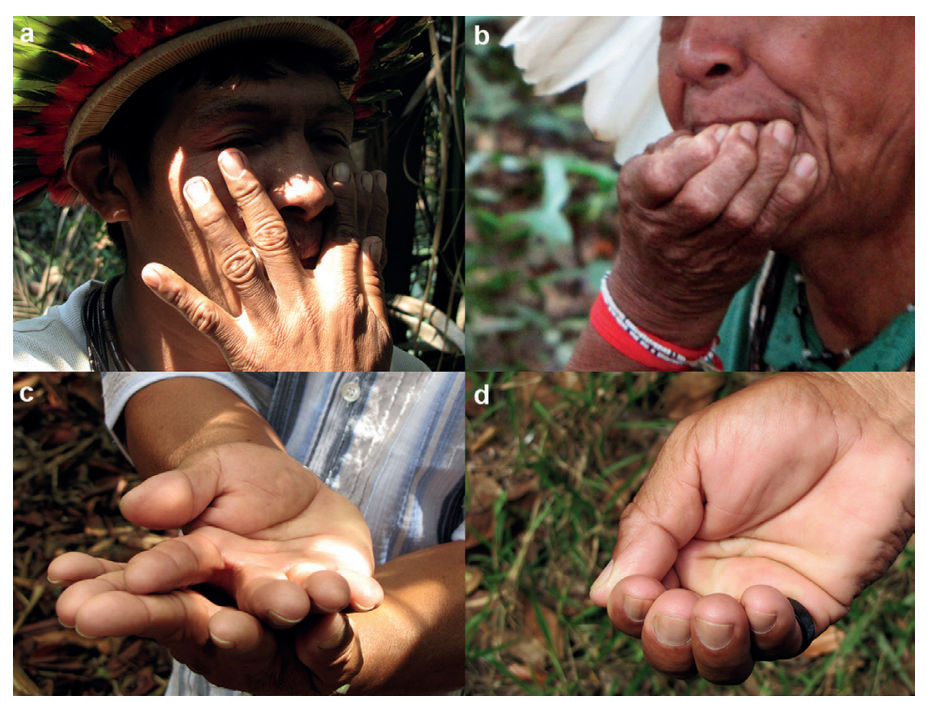

Figura 3. - Técnicas de la población Gavião de Rondônia, en la Amazonía brasileña, para silbar con resonancia en los manos.

Fotos $\boldsymbol{a}$ y $\boldsymbol{b}$ reproducidas de Moore y Meyer (2014).

Fotos $\boldsymbol{c}$ y $\boldsymbol{d}$ reproducidas de Meyer (2012). 


\section{Diversidad de las lenguas en cuestión}

Los lenguajes silbados basados en idiomas no presentan silbos que signifiquen por sí solos conceptos como «cabra»o «casa», sino que se requiere la combinación de elementos lingüísticos silbados y articulados para formar palabras que a su vez se combinan en oraciones, como sucede en la lengua hablada, ya que los silbidos imitan muchos aspectos de las propiedades lingüísticos del habla. Esto permite a sus practicantes emitir cualquier tipo de mensaje que no haya sido convenido previamente.

El lenguaje silbado es una práctica poco conocida porque no siempre fue identificada como un fenómeno lingüístico por los investigadores y porque pierde rápidamente vitalidad con la modernización de la vida rural, aunque se dé en una amplia gama de lenguas y familias lingüísticas en todos los continentes poblados del planeta (fig. 4 y tabla 1). Según referencias científicas, han sido hallados en el mundo más de setenta idiomas con lenguaje silbado. Cerca de cuarenta de ellos han sido estudiados o grabados en su forma silbada en particular (Meyer, 2015). Los idiomas que presentan modalidad silbada existen en familias lingüísticas tan diferentes como la Afro-Asiática y Níger-Congo, en África; Algonquiana, EsquimoAleutiana y Oto-Mangueana en América Central y America del Norte; Arawak, Jê y Tupí en America del Sur; Altaica, Indo-Europea y SinoTibetana en Eurasia; y Torricelli, Trans-Nueva Guineana y Sepik, en Oceanía. Esta diversidad lingüística muestra que esta práctica no está limitada a un tipo de estructura lingüística o a un tipo de familia lingüística. Más bien al contrario, sugiere que todas las lenguas humanas pueden desarrollar una forma silbada si las condiciones ecológicas y sociales lo requieren. Además, se desarrolló tanto para idiomas tonales (como el mixteco y el chinanteco en México, o akha y hmong en el Sudeste Asiático) como para idiomas no tonales (como el griego, el español, el turco, el bearnés, el wayãpi o el tamazight) e incluso para un caso raro de idioma con tonos nacientes (lengua chepang, del sur de Nepal). 


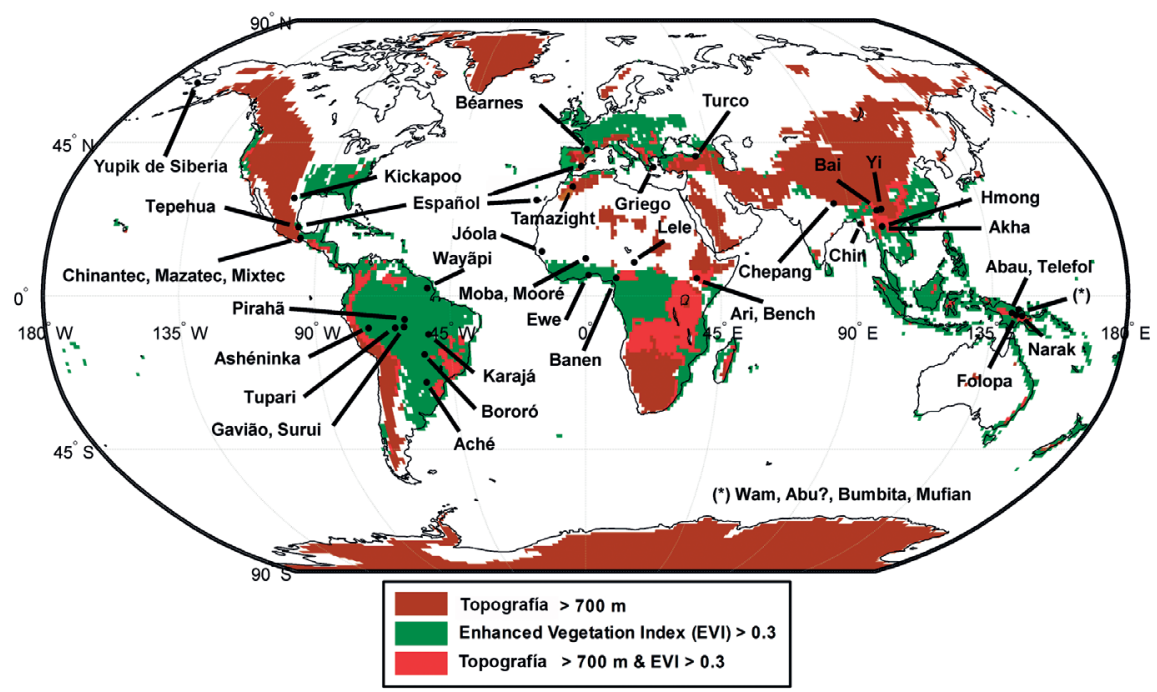

Figura 4. - Mapa de las lenguas del mundo con una forma silbada comprobada, incluyendo indicaciones de factores ambientales asociados a paisajes de montañas o de bosques densos (topografía, índices de vegetación).

Fuentes para topografía: AIRS + ASMU, GES DISC.

Fuente para vegetación: MODIS/Terra Monthly Vegetation Indices Global 1*1 degree, NASA NEESPI data and service center.

\begin{tabular}{|l|l|}
\hline Región del mundo & Lenguas \\
\hline África & $\begin{array}{l}\text { attie, bafia, baïnuk, bape, birifor, bobo, burunsi, doohwayoo, } \\
\text { gbaya, gurma, lobi, kabye, kasem, manjack, marka, mofu, } \\
\text { mwana, nchumburu, tshi, ule, wobe, yacouba, yoruba }\end{array}$ \\
\hline América Central & amuzgo, chol, otomí, Sayula popoluca, totonac, zapotec \\
\hline América del Norte & taos \\
\hline América del Sur & desano, makurap, siriono, timbira \\
\hline Oceanía & $\begin{array}{l}\text { aruek, au, bauzi, binumarien, gadsup, palai, tairora (en Papúa } \\
\text { Nueva Guinea); punan busang (en Sarawak, Malasia) }\end{array}$ \\
\hline
\end{tabular}

Tabla 1. - Lenguas con lenguaje silbado señaladas en publicaciones científicas (sólo referencias, sin que haya sido estudiado el funcionamiento de la práctica), según Meyer (2015).

De investigaciones previas se sabe que el habla silbada modifica profundamente la fonética del habla modal, el habla con voz, aplicando una reducción en el dominio de la frecuencia: transformando el complejo espectro de frecuencia de la voz en una imitación basada en una variación sencilla del tono del silbido (fig. 5 y 6). Durante este procedimiento, ciertos detalles 
fonéticos presentes en el habla modal se pierden: al utilizarse únicamente la parte anterior de la boca, se reduce la precisión con respecto a la lengua hablada, lo que provoca frecuentes casos de ambigüedad en la recepción de los mensajes silbados. Los interlocutores resuelven esta carencia gracias al contexto de la situación o por medio de preguntas aclaratorias. No obstante, pueden ser observados altos niveles de inteligibilidad, en particular en las lenguas no tonales o en las lenguas tonales con un alto número de tonos fonológicos distintivos (Busnel, 1970; Rialland, 2005; Meyer, 2015; Sicoli, 2016). Al comparar las versiones silbadas de varios idiomas del mundo, se observa que cada una se adapta a varios aspectos de la estructura de la lengua que se está sustituyendo. Sin embargo, se produce una diferencia importante en la estrategia de sustitución entre las lenguas tonales (fig. 6) con respecto a las no tonales (fig. 5). Lo que diferencia las lenguas tonales de lenguas no tonales es que en los idiomas tonales, la pronunciación de los tonos influye en el significado de las palabras, cosa que no ocurre en las lenguas no tonales.

En la mayoría de las lenguas no tonales los silbadores se aproximan a la articulación del aparato vocal utilizado en la forma hablada; este enfoque provoca una adaptación silbada de la cualidad de las vocales y consonantes realizadas por el timbre de la voz. Por ejemplo, en griego, español y turco, las vocales silbadas son emitidas en los diferentes niveles de tono en función de la distribución de frecuencias de su timbre (en particular por las zonas de resonancia principales llamadas «formantes»): normalmente, /i/ tiene el tono más alto, /e/ es menor, /a/ es incluso menor, y /o/ tiene el tono más grave (Meyer, 2005, 2008; Díaz Reyes, 2008) como ilustran las fig. 5 y la fig. 12. Ha sido demostrado recientemente que este tipo de distribución de vocales silbadas en una escala de frecuencias simples corresponde a una realidad perceptiva que permite discriminar las vocales entre ellas no solamente para silbadores competentes en un lenguaje silbado, sino también para oyentes sin ningún conocimiento sobre este fenómeno (Meyer, Gautheron \& Ridouane, 2015; Meyer, Dentel \& Meunier, 2017). También ha sido demostrado que los individuos se adaptan tanto en producción (Meyer, 2015) como en percepción (Meyer, Dentel \& Meunier, 2017) a las especificidades de pronunciación de cada vocal en cada lengua. Las consonantes están representadas por modulaciones continuas o interrumpidas de estas frecuencias vocálicas, cuando están entre dos vocales (Leroy, 1970; Busnel \& Classe, 1976; Trujillo, 1978; Rialland, 2005). Se realizan imitando lo mejor posible su articulación y su sonido en la lengua hablada. La descripción del mecanismo de la producción de silbidos bilabiales ha mostrado que una frecuencia de silbo siempre está capturada por el segundo o tercer formante del aparato vocal, y que se produce un salto de 
frecuencia entre los dos cuando estos formantes están cerca (Shadle, 1983). Este último efecto ocurre en vocales anteriores (como la /i/) y en algunas transiciones de consonantes. Esta importancia de otros formantes, como el segundo, fue confirmada con la descripción detallada del sistema vocálico de lenguas con muchas vocales, como el caso del turco (Meyer, 2015).

En las lenguas tonales (ver Rialland, 2005 y Meyer, 2015 para una revisión), en lo que se llama silbido basado en el tono, la situación es diferente: los silbidos no se centran en imitar el timbre, sino el tono de la voz, trasponiendo la frecuencia fundamental de las cuerdas vocales para codificar principalmente los tonos léxicos. Por lo tanto, en la forma silbada de una lengua tonal, la cualidad vocal está completamente excluida. Esta exclusión de timbre se produce incluso cuando la carga funcional de información transportada por tonos es menor que la correspondiente a la cualidad vocal.

a

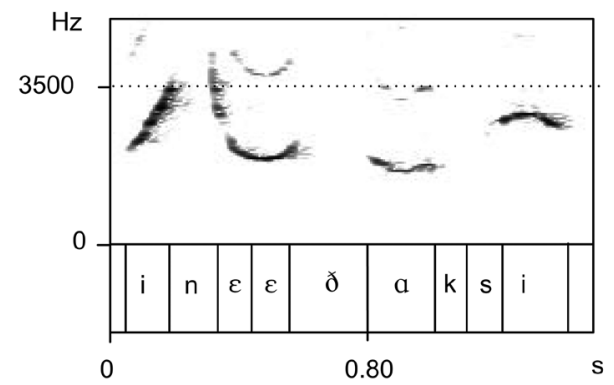

b

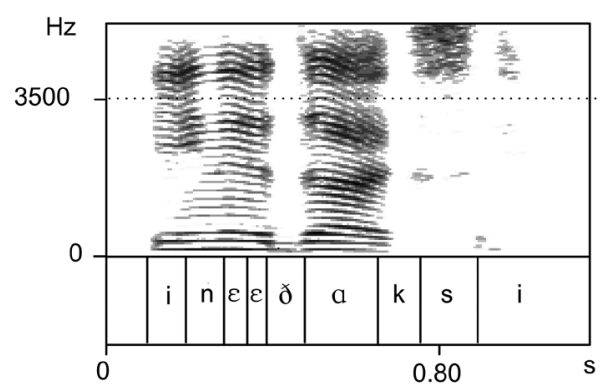

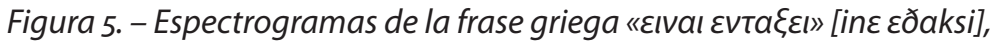
0 «ine entaksi» en transliteración romana (significando «todo bien») silbado (a) y hablado (b).

Aquí, la forma silbada de esta lengua no tonal consiste en la emulación de las cualidades de las vocales y de las consonantes (que están principalmente caracterizadas acústicamente por los formantes). 
a

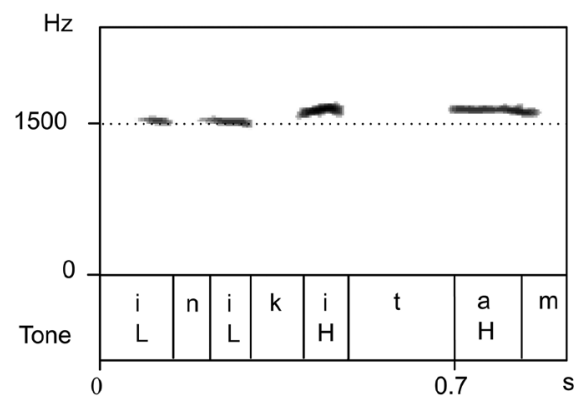

b

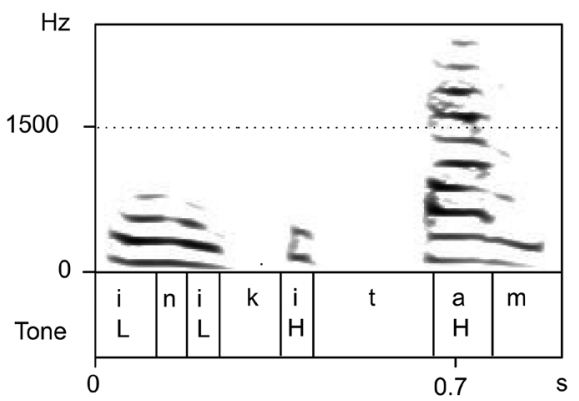

Figura 6. - Espectrogramas de las palabras gavião [ini kitam], que significan "cuerda de hamaca», silbado (a) y hablado (b).

Aquí, la forma silbada consiste en la emulación del tono («pitch» en inglés) de la forma hablada.

Por tanto, la versión silbada se adapta a cada idioma y a grandes distinciones tipológicas: los silbadores adaptan sus silbidos al número de tonos en la lengua o al número de vocales, y cómo los tonos y las vocales se articulan con las consonantes (ver Busnel \& Classe, 1976; Meyer, 2015 para mas detalles sobre todas lenguas, y Classe, 1957 o Díaz Reyes, 2008 para datos mas precisos sobre el español).

La estructura acústica de las vocales habladas y su mecanismo de producción permite explicar la distinción tipológica entre lenguas tonales y lenguas no tonales en las estrategias de imitaciones silbadas: la producción vocal hablada se caracteriza tanto por una frecuencia fundamental clara y fuente del sonido que resulta de la vibración de las cuerdas vocales en el fondo de la laringe (llamada F0, y que codifica el tono léxico cuando sucede en lenguas tonales, y también la acentuación o la entonación en varias lenguas) y una clara estructura resonante del tracto vocal por completo (los formantes que organizan el espacio vocálico de cada lengua, por ejemplo). Estos dos niveles de frecuencia son controlados en gran parte 
independientemente (Fant, 1960), y pueden funcionar fonológicamente en la mente humana como canales separados de información para codificar el habla porque también son recuperables independientemente y de manera perceptual a través del tono (basado en el F0) y del timbre (basado en el espectro completo de la voz, incluyendo los formantes) (Risset, 1968). En este sentido, la voz hablada contrasta con una forma silbada del habla, en la que no es posible, en un silbido, controlar por separado el F0 y la resonancia, ya que son producidos juntos en el tracto vocal. De hecho, al silbar, el F0 del silbo es también la resonancia silbada en el tracto vocal. En la mayoría de los idiomas, los silbadores resuelven este dilema al elegir emular solo uno de los dos atributos perceptivos que se llevan en el habla normal (tono o timbre) y adaptarlo a la fonética de su lenguaje, como acabamos de describir. En la mayoría de las lenguas no tonales no hay mucha concurrencia entre los dos niveles perceptivos porque el tono no carga mucha información léxica, como sí sucede en las lenguas tonales.

\section{Un antiguo sistema de telecomunicación asociado a ciertos entornos ecológicos y ciertos contextos rurales tradicionales}

Es notable que las formas silbadas de idiomas que se observan en el mundo se han desarrollado en contextos ecológicos particulares: se encuentran sobre todo en los paisajes montañosos y con alta densidad de vegetación (fig. 1, 2, 3, 6, 7 y 8). Es uno de los aspectos más llamativos de su distribución a nivel mundial: darse casi exclusivamente en asociación con ciertos tipos de hábitats. Este fenómeno lingüístico especial y complementario del habla sobrevive en poblaciones remotas y de baja densidad, porque tiene la ventaja de aumentar el rango audible de expresión y permite diálogos cuando los hablantes están alejados unos de otros. Efectivamente, todas las zonas donde los lenguajes silbados han sido registrados tienen la característica de crear una demanda de comunicación a larga distancia debido a su densa vegetación y/o topografía; como resultado de aislar rápida y frecuentemente a los individuos, y restringir su comunicación oral normal o gritada. En varias situaciones, asociadas principalmente a entornos naturales rurales, puede ser muy útil comunicarse a larga distancia con toda la riqueza comunicativa de un idioma. Por ejemplo, para señalar un peligro o dar noticias importantes a alguien situado del otro lado de un valle o a un interlocutor difícil de localizar porque está escondido por un bosque, o en su casa. Gritar es una forma de adaptación del habla que fue adoptada universalmente para este tipo de situaciones. Pero el grito humano está limitado en distancia y en tiempo de dialogo porque las cuerdas vocales se 
cansan y dañan con el esfuerzo. En cambio, la producción de silbidos no hace sufrir las cuerdas vocales porque no las utiliza en el modo vibrante, sino solo para controlar la fuerza de la corriente de aire. Además, los interlocutores distanciados necesitan señales potentes y claras, poco perturbadas por el ruido ambiente natural y los obstáculosfísicos que afecten a la propagación del sonido. Las características físicas de los silbidos estánbien adaptadas a estas limitaciones ambientales porque permiten mucha potencia en una gamaestrecha de frecuencias (1000 a 4000 Hertzios) que corresponde a la mejor audibilidad y discriminación sonora del ser humano y que es más alta que las frecuencias más potentes del ruido de fondo de la naturaleza. Por eso, un silbido llega mucho más lejos que un grito y así evita que los interlocutores viajen largos trayectos para hablar entre sí (ver por ejemplo fig. 7 y 8 ).

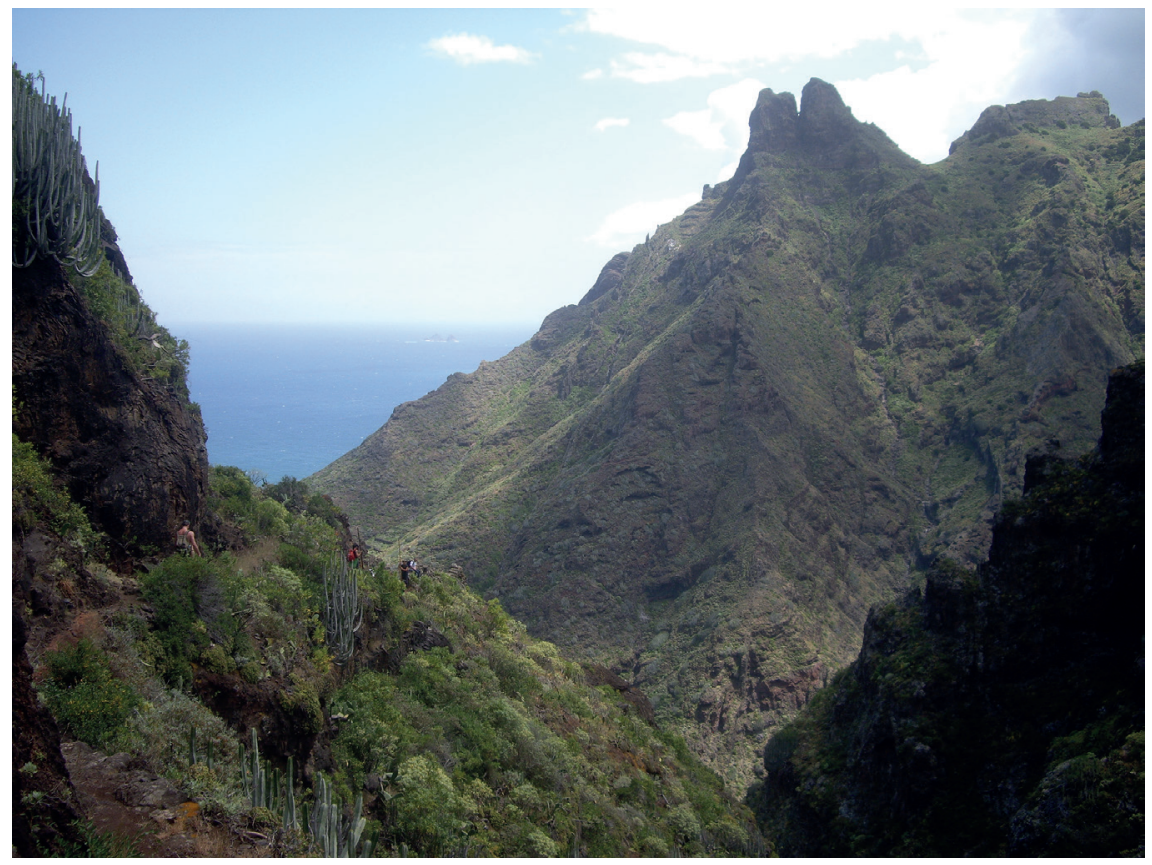

Figura 7. - Montañas de Anaga, Tenerife, Islas Canarias.

(c) David Díaz Reyes. Todos los derechos reservados.

En los bosques tropicales densos como el Amazonas, las frecuencias de los silbidos son muy adecuadas para hacer frente a las pérdidas de energía sonora que sufre una señal acústica cuando se refleja en las plantas que actúan como barrera (fenómeno de reverberación debido a las hojas y 
troncos). En este tipo de ambiente, el lenguaje silbado facilita la coordinación de los individuos durante los movimientos en grupo, especialmente durante la caza (fig. 8). Permite a los interlocutores localizarse y discutir cuando el contacto visual se pierde rápidamente debido a la densidad de la vegetación. También tiene la ventaja de fundirse con los ruidos de la naturaleza y, por lo tanto, ser difícil de detectar por los animales, ya que muchos de ellos también utilizan sonidos silbados. Por lo tanto, es un activo real para acercarse a la presa durante la caza. En un bosque denso, la comunicación silbada se utiliza a partir de más o menos 10 metros y hasta aproximadamente 500 metros, dependiendo de la densidad de vegetación.

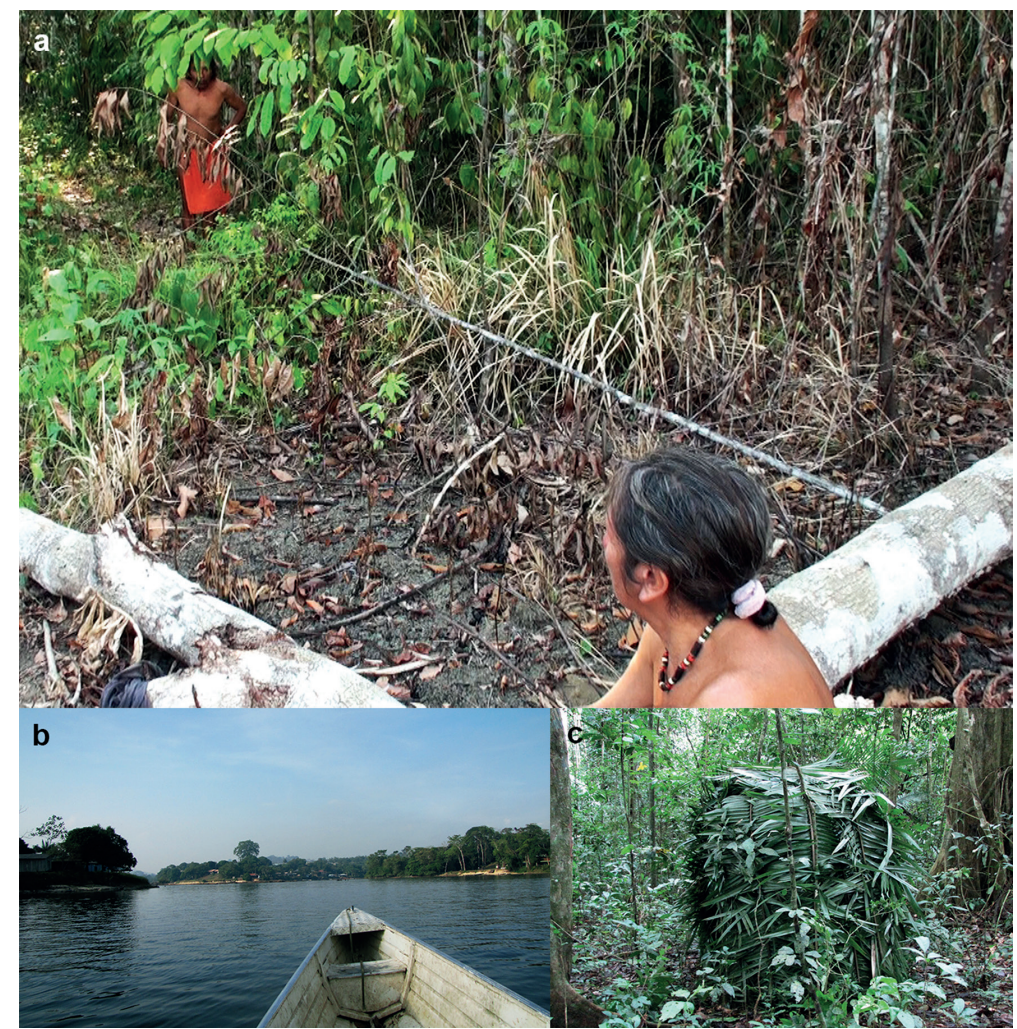

Figura 8. - a. Silbadores Wayãpi usando la tecnica bilabial en Guiana Francesa; b. Ejemplo de paisaje típico encontrado en un pueblo situado cerca de un río de la selva amazónica; c. Ejemplo de un pequeño refugio donde los Gavião se esconden para cazar.

Fotos: cortesía de Julien Meyer. (C) Julien Meyer. Todos los derechos reservados. 
En las montañas, el tiempo de caminata entre dos puntos distanciados puede ser largo y difícil, mientras que la topografía es favorable para el contacto visual y la propagación del sonido (fig. 7 y 9). Los valles crean guías de ondas, mientras que las diferencias de altitud contribuyen a una buena propagación de la señal. El lenguaje silbado evita largas horas de caminata en relieves escarpados y, además, hace posible entrar en contacto y dialogar con un individuo que se ve de lejos o cuya localización se conoce. En las montañas, la forma silbada de un idioma sirve tan pronto como la forma hablada se vuelve ineficaz; entre 40 y 100 metros, dependiendo del terreno. Se puede oír hasta varios kilómetros de distancia en algunos valles. En los Pirineos, las distancias de uso del bearnés silbado era hasta 2 kilómetros (Busnel, 1964). En las Islas Canarias, los valles empinados permiten que los silbadores alcancen registros de distancia, ya que constituyen auténticas guías de ondas que llevan la señal hasta, en ocasiones, cerca de 8 kilómetros (Busnel \& Classe, 1976).

En general, un lenguaje silbado está anclado en comunidades relativamente poco pobladas. Es facilitador de la organización del día a día y, a veces, participa en la transmisión de ciertos aspectos de la tradición oral. Permite a los miembros de un grupo (familia, pueblo) coordinar sus actividades de subsistencia como caza, pesca, cultivos en zonas montañosas, o custodia de ganado por pastores. También permite transmitir noticias, o anunciar una emergencia. Es un medio de comunicación adecuado en condiciones meteorológicas difíciles (niebla o calima densa, lluvia...) o por la noche. También lo es para hablar en secreto en presencia de extraños. Por ejemplo, en los Pirineos durante la Segunda Guerra Mundial lo fue para evitar el impuesto de guerra (Arripe, 1985), o en varias Islas Canarias para organizar la resistencia a los conquistadores (Marín de Cubas, 1694; Bethencourt Afonso, 1912; Díaz Reyes, 2008). Otro ejemplo ocurrió en Papúa Nueva Guinea, durante la Segunda Guerra Mundial, cuando silbadores Wam fueron reclutados por los australianos para engañar a los japoneses que escuchaban sus comunicaciones (Nekitel, 1992).

En algunos lugares también sirve para acompañar otros momentos importantes de la vida (nacimiento, seducción, matrimonio, defunciones, festivales), particularmente en el sudeste asiático donde se ha comprobado su uso para poesías amorosas (seducción y matrimonio), en ocasiones siendo esta la principal actividad en la cual sobrevive el lenguaje silbado (Meyer, 2015).

Dependiendo de la distancia a alcanzar, se utilizan diferentes técnicas para crear un chorro de aire en vibración que resuena en la boca (fig. 1,2, 3 y 9): ya sea avanzando los dos labios - técnica bilabial - para comunicarse a distancias relativamente cortas (hasta unos 50 metros) o girando la 
punta de la lengua contra los dientes inferiores - técnica linguo-dentallo que crea un chorro más potente, o mediante la introducción de uno o dos dedos para fortalecer aún más la presión del aire, permitiendo alcanzar varios kilómetros cuando las condiciones meteorológicas y topográficas son favorables en las montañas.

En cada lugar, las técnicas más populares para producir silbidos asociados a lenguajes silbados están relacionadas con las actividades dominantes de subsistencia (pastoreo, caza, pesca, cultivos de montaña) que dependen del paisaje y de las distancias de comunicación que se les supone. Así, en la selva amazónica se ha observado principalmente la técnica bilabial y técnicas con una/las mano/s resonante/s enfrente de la boca para bajar un poco la frecuencia del silbido (fig. 3), lo que garantiza una mejor propagación en biotopos caracterizados por vegetación densa. En montañas son las técnicas linguo-dental o con dedos introducidos en la boca que dominan porque son más potentes, necesarias para alcanzar comunicaciones de un lado al otro de los valles.

Estas observaciones ofrecen perspectivas interesantes porque sugieren que un lenguaje silbado está fuertemente estructurado por diferentes nichos ecológicos y por cómo las actividades humanas de subsistencia se adaptan a los biotopos locales. Las características naturales del entorno parecen también representar, al menos, una de las condiciones necesarias de la existencia de sistemas de comunicación de habla silbada, si no su invención. Por lo tanto, la observación de los lenguajes silbados del mundo subraya que la información y la comunicación, que desempeñan papeles importantes como motores de la evolución cultural en las sociedades modernas (Finke, 2005, 2006), pueden haber sido a veces profundamente afectadas a lo largo de la historia por los contextos ambientales en las pequeñas sociedades rurales. Este aspecto geolingüístico, que ha sido poco abordado hasta la fecha en los trabajos sobre ecología humana (Meyer, 2015) es interesante para señalar la importancia de los ecosistemas en la evolución del lenguaje humano, lo que hasta ahora había sido investigado principalmente en los contenidos léxicos de los idiomas, que son claramente influenciados por los entornos ecológicos de los hablantes.

\section{Ejemplo de la lengua española: Canarias y otros lugares}

\subsection{El Silbo en las Islas Canarias}

El Archipiélago Canario es uno de los lugares del mundo donde las poblaciones humanas han sabido convertir las modulaciones del silbo en palabras inteligibles, con el fin de emitir y recibir mensajes a larga distancia. El 
silbo es el sonido más intenso que una persona puede producir sin utilizar elementos ajenos a su propio cuerpo. Esto es especialmente útil en sociedades rurales de Canarias que habitan espacios montañosos, a veces con vegetación densa, por la dificultad que supone cubrir, en estos casos, las distancias de comunicación para la realización efectiva de actividades de supervivencia como el pastoreo o los cultivos en montañas.

Los primeros pobladores de esta región del mundo eran bereberes llamados comúnmente los Guanches, y eran relacionados con el noroeste de África, donde existe constancia histórica y actual de uso de idiomas bereberes silbados en diversas zonas montañosas de Marruecos (Pucheu, 2015; Meyer, 2015; Meyer, Gautheron \& Ridouane, 2015) ${ }^{1}$. Además, está perfectamente documentado que al menos algunas poblaciones indígenas de las islas, hablantes de lenguas de origen bereber, practicaban el lenguaje silbado al momento de la conquista europea (Espinosa, 1594; Abréu Galindo, 1632; Marín de Cubas, 1694). Una posible primera prueba de la existencia de una forma silbada de un idioma de las islas Canarias en la época prehispánica se encuentra en el testimonio escrito de dos pastores franciscanos que acompañaban al mercenario galo Jean de Bethencourt en 1402 (Bontier \& Le Verrier, 1630, ver extracto de este diario en Busnel \& Classe, 1976 y en Meyer, 2015). Otros testimonios antiguos como los de Fray Alonso de Espinosa (1594) o textos de análisis histórico o antropológico (Chil \& Naranjo, 1880; Quedenfeldt, 1887; Verneau, 1891) explicaran que las formas silbadas de esta(s) lengua(s) bereber(es) se utilizaban al momento de la conquista y del inicio de la colonización para comunicación a larga distancia en las islas de La Gomera, El Hierro, Tenerife y Gran Canaria por los habitantes autóctonos, llamados comúnmente guanches. Por falta de datos lingüísticos asociados a los guanches, no se sabe si ellos hablaban diferente dialectos de un mismo idioma o si hablaban diferentes idiomas, pero se conoce que estos fueron hablados hasta el siglo XVI o XVII.

1. Gerard Pucheu, después de un trabajo de investigación propio iniciado con los consejos de Busnel, Meyer y Gautheron, viajó en 2013 para el Alto Atlas y finalmente localizó varios silbadores competentes en la lengua bereber tamazight. En 2014 y 2015, Meyer, Gautheron y el lingüista Bereber Rachid Ridouane realizaran dos estadías en el campo para documentar e investigar este fenómeno. Estas tres publicaciones son posteriores a la comunicación de Laure Dentel, en 2006, sobre el hecho de que había, en el libro Désert (Le Clézio, 1980), una descripción de un dialogo en frases silbadas en el idioma tashelhit bereber hablado en Marruecos. Esta información motivo los contactos de Dentel (René-Guy Busnel, Julien Meyer, Bernard Gautheron) a buscar informantes que podrían ayudarles a localizar locutores en las montañas del Atlas y en el desierto. 
Durante el siglo XV y XVI estas islas fueron colonizadas principalmente por andaluces, castellanos y portugueses, que dominaron y, en algunos casos, eliminaron las poblaciones nativas guanches. Estas fueran gradualmente asimiladas y sus idiomas o dialectos se extinguieron. No es de extrañar, por tanto, que la población canaria resultante tras el mestizaje que provocó la conquista y colonización por parte de la Corona de Castilla (oficialmente, de 1402 a 1496 d.C.) siguiera haciendo uso del lenguaje silbado, simplemente porque le era útil en sus actividades cotidianas. La lengua que se sustituyó al silbar ya no fue jamás un idioma de origen bereber, sino una de origen románico: el español o castellano. Esta traslación habría sucedido, con toda probabilidad, entre los siglos XV y XVI, puede que incluso hasta el XVII, dependiendo de las zonas. Según sus testimonios, cuando Quedenfeldt, Lajard o Verneau visitaran las Islas Canarias a finales del siglo XIX, la forma silbada de la lengua local ya era en español. Como las lenguas bereberes y español, tienen en común que son no tonales, este hecho habría simplificado el traslado de una para a otra.

Como los otros lenguajes silbados que proceden de idiomas no tonales del mundo, el lenguaje silbado encontrado en las Islas Canarias es un lenguaje completamente sustitutivo del hablado, en este caso, del español hablado en cada isla, y del que posee su estructura básica. El mensaje es emitido, de media, a una intensidad potente, algo superior a los 100 decibelios. Dependiendo de factores como el silbador, la orografía, la cantidad de vegetación, la altura sobre el nivel del mar, el tipo de terreno, el ruido y hasta de agentes meteorológicos, se puede escuchar y entender con relativa facilidad el mensaje silbado a distancias de uno o dos kilómetros, siendo posible, en caso de tener todas las circunstancias favorables, alcanzar y hasta superar los tres. En la naturaleza del silbo utilizado en Canarias está trasmitir lo público, no lo privado, aunque en ocasiones se haya utilizado para no ser entendido por extranjeros llegados a las Islas. Al tratarse de un sistema de comunicación a distancia, las personas que tradicionalmente lo han utilizado son pastores, agricultores, cazadores...; personas que se ven frecuentemente aisladas en terrenos agrestes y distanciadas entre sí. Este tipo de actividades explica que la utilización haya sido principalmente masculina, probablemente en torno al $80 \%$, frente al $20 \%$ de mujeres, como han reflejado nuestras investigaciones. 


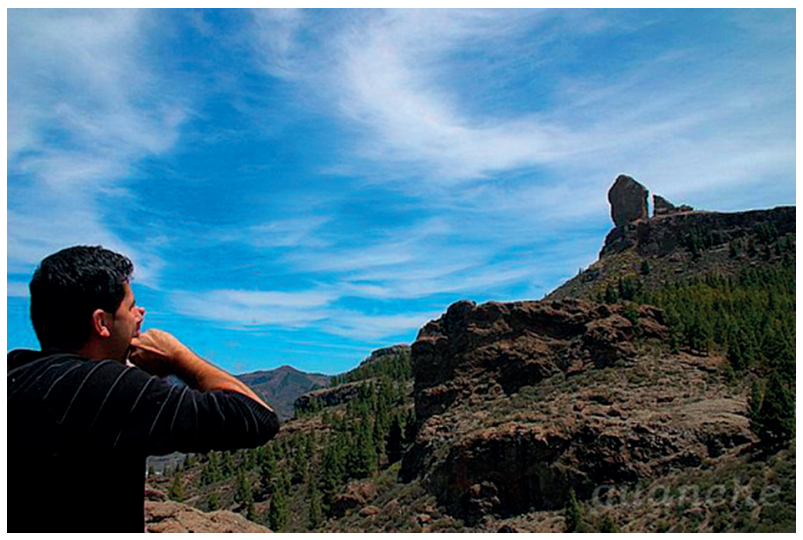

Figura 9. - Silbando en el centro de la isla de Gran Canaria. Imagen: cortesía de Jorge Videgaín. (C) Jorge Videgaín. Todos los derechos reservados.

Como breve indicación, pasamos a comentar aquí la situación y distribución pasada y presente del lenguaje silbado español en el Archipiélago Canario (fig. 10):

- LA GOMERA: lo conservó con cierta vitalidad hasta nuestros días, por diferentes motivos. Con fuerza, hasta los años 70 del siglo Xx, siendo la única isla donde el lenguaje silbado es actualmente aprendido en la enseñanza pública de manera reglada, además de otras iniciativas particulares. Es conocido y practicado por buena parte de su población. La Gomera es la isla del silbo por antonomasia, la que más estudios ha cosechado y la que le ha dado el conocido nombre de silbo gomero.

- EL HIERRO: lo conservó con vigor hasta los años 60 del siglo xx, siendo escaso su uso posteriormente (Díaz Reyes, 2008, 2017). Aún quedan numerosos silbadores vivos, la mayoría son de avanzada edad, excepto aquellos de un aprendizaje reciente. Existe buena documentación gráfica (Lajard, 1891; Ricard, 1930; Trapero, 1991; Lorenzo Perera, 2002) y etnográfica de su utilización, y su revalorización está empezando a ser efectiva por parte de las instituciones locales (Cabildo Insular de El Hierro) e iniciativas particulares.

- TENERIFE: lo conservó al menos hasta los años 40 del siglo xx. Está ampliamente documentado (Espinosa, 1594; Abréu Galindo, 1632; Glas, 1764; Bethencourt Alfonso, 1912; López Soler, 1922), y aún hoy viven algunos silbadores de tradición que, aunque no lo usan, son capaces de emitir y comprender mensajes silbados (Díaz 
Reyes, 2011). Además, en la actualidad cientos de personas lo usan gracias a un aprendizaje reciente.

- GRAN CANARIA: lo conservó con vigor al menos hasta los años 40 del siglo Xx. Su uso ha sido documentado (Agrupación Etnográfica La Aldea, 1992; Perera Mendoza, 2009; Jiménez Medina et al., 2012; Quesada, 2017) y en un estudio etnográfico reciente hemos encontrado algunos buenos silbadores, lo que ha motivado la presentación de la ponencia El lenguaje silbado en la Isla de Gran Canaria en el V Encuentro con el Lenguaje Silbado que organiza la Asociación Yo Silbo. Actualmente, cientos de personas lo usan tras un aprendizaje reciente.

- LANZAROTE: su uso no ha sido confirmado. No obstante, existe una leyenda relacionada con el lenguaje silbado que gira en torno a la Peña del Silbo, zona montañosa entre Teguise y Haría, además de pistas sobre su uso pasado en los Ajaches y otras zonas de la isla (Díaz Reyes, 2011).

- FUERTEVENTURA: su uso no ha sido confirmado. También existe un topónimo, la Montaña del Silbo, en La Oliva, y el testimonio de personas que comentan sobre la presencia de pastores silbadores llegados a la isla desde El Hierro.

- LA PALMA: su uso no ha sido confirmado. Sin embargo, en entrevistas de trabajo de campo, que aún continúan, hemos sido informados de un uso pretérito entre pastores de La Caldera de Taburiente $\mathrm{y}$ el noroeste de la isla.

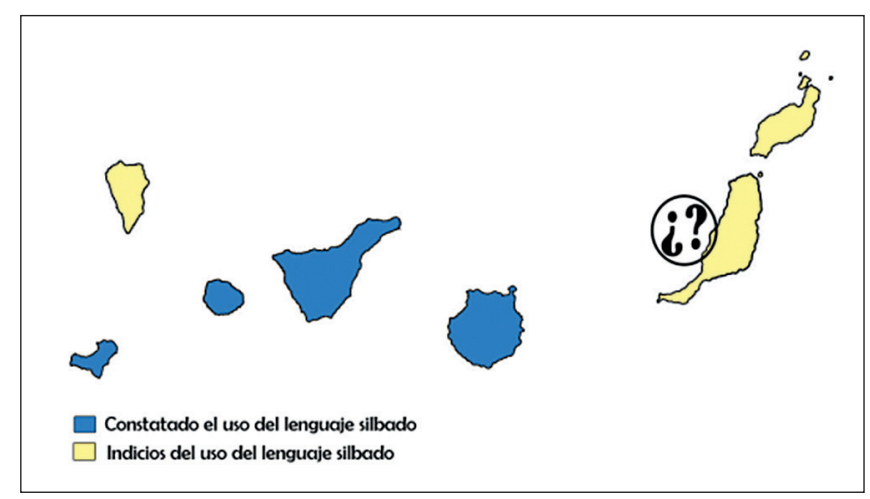

Figura 10. - Islas Canarias. Uso de lenguaje silbado.

Imagen: cortesía de Guillermo Molina.

(c) Guillermo Molina. Todos los derechos reservados. 


\subsection{Español silbado en otras zonas del mundo}

El silbo español de La Gomera, una de las Islas Canarias, localmente llamado «Silbo» o «El Silbo», es el más estudiado de todos los lenguajes del mundo, y posee una gran importancia histórica y cultural. Este hecho, junto con su grado de conservación y las medidas de revalorización que han sido llevadas a cabo por las instituciones públicas le han valido la declaración de Obra Maestra del Patrimonio Oral e Inmaterial de la Humanidad por la UNESCO en 2009. No obstante, el silbo en español no es exclusivo de La Gomera, ni de las otras Islas Canarias, pues existen otros lugares del mundo donde se silba o se ha silbado en esta lengua.

En México, a más de 8000 kilómetros de Canarias, podemos hallar comunicación silbada en español. Son varios los investigadores que han hecho referencias a esta práctica, pero pocos los que han llegado a un análisis lingüístico. El geógrafo Schmieder, referido a los indios zapotecas y los mixes del estado de Oaxaca, citó en 1930:

[...] una singular práctica de los habitantes de la montañas, totalmente desconocida en el valle, es el chiflo o lenguaje silbado. El chiflo imita el lenguaje hablado y los pobladores de la sierra son capaces de silbar tanto en español como en zapoteca. Mediante el silbo se llaman unos a otros por el nombre y mantienen conversaciones sencillas. Es frecuente oír a los hombres de las montañas, al igual que los niños cuando juegan, silbándose de esta manera.

El investigador Juan A. Hasler hace referencia al silbo en español entre los cafetaleros de Huatusco, municipio de la zona montañosa central del estado de Veracruz, y en Bermúdez, localidad de canteros del municipio de Huaxca. Él apuntó también silbidos en español en la Región mexicana de Tlaxcala, donde la práctica había sido notada en el municipio de Juan Cuamatzi (Hasler, 1960) y más tarde se escuchó en gran parte del sur de la región, según Wilken (1979). Según estos autores, los silbadores de Tlaxcala usaban dientes, lengua y dedos para silbar, como en La Gomera. El único análisis lingüístico de una práctica de español silbado en Mexico fue realizado por la investigadora Coberly (1975) con grabaciones realizadas por Wilken en el municipio de San Juan Zacualpan. Ella realizo un análisis comparativo de vocales y consonantes silbadas de La Gomera y de Tlaxcala. Lo interesante de esta comparación es que demuestra que en Tlaxcala las vocales y las consonantes silbadas tienen el mismo aspecto que en La Gomera, pero no encontró evidencia clara de transferencia de técnica silbada entre estas dos culturas que silban el español. Por eso, el misterio permanece completo sobre el origen de esta práctica en Tlaxcala y otros lugares de México: ¿Sera que llegó con los colonos españoles que 
transitaban por las Islas Canarias? ¿O fue transferido desde una lengua prehispánica al español a través de una práctica tradicional local preexistente? Efectivamente, el lenguaje silbado es común entre varios grupos indígenas de México, bien sea para sustituir una lengua tonal, como el mazateco, el zapoteco, o el chinanteco, o una lengua no tonal como el totonaco o el tepehua (Cowan, 1976). Está por investigar cuánto interviene este aspecto en la sustitución de la lengua que llevaron a México los conquistadores europeos, el español.

Otro lugar del mundo en que se ha comunicado con silbidos en castellano lo hallamos en la propia España, concretamente en, por lo menos, varios pequeños pueblos de la provincia andaluza de Almería llamados Topares, Cañada de Canepla, Desdre y Puebla de Don Fadrique. En 2014 hallamos, al menos, dos pastores de la localidad de Topares que aún conocían cómo hacer un pequeño silbado de barro cocido llamado pito o boca (fig. 11) y cómo hablar en español (fig. 12) con esta herramienta (Meyer, 2015). En el pasado lo usaron mientras trabajaban para comunicarse entre ellos en español silbado, y para dar órdenes a sus perros u ovejas. En una primera investigación, de 1992, sobre este fenómeno, hecha por el Centro de Documentación Musical de Andalucía, se decía que la práctica era residual, pero que algunos pastores mayores de 50 años de varias villas de la misma región eran aún capaces de explicar y demostrar esta técnica, y haciendo siempre referencia a su uso en actividades y labores pastoriles (Cañadas Asencio \& Jiménez Morales, 1992). Hoy en día, la práctica está casi perdida, existiendo únicamente en el recuerdo de unos pocos ancianos silbadores. Es muy complicado sugerir el origen del uso de lenguaje silbado en esta región por el hecho de que hubo frecuentes conexiones históricas tanto con el norte de África como con toda la región mediterránea y con las Islas Canarias, además de que este tipo de pito para hablar es único en el mundo. 


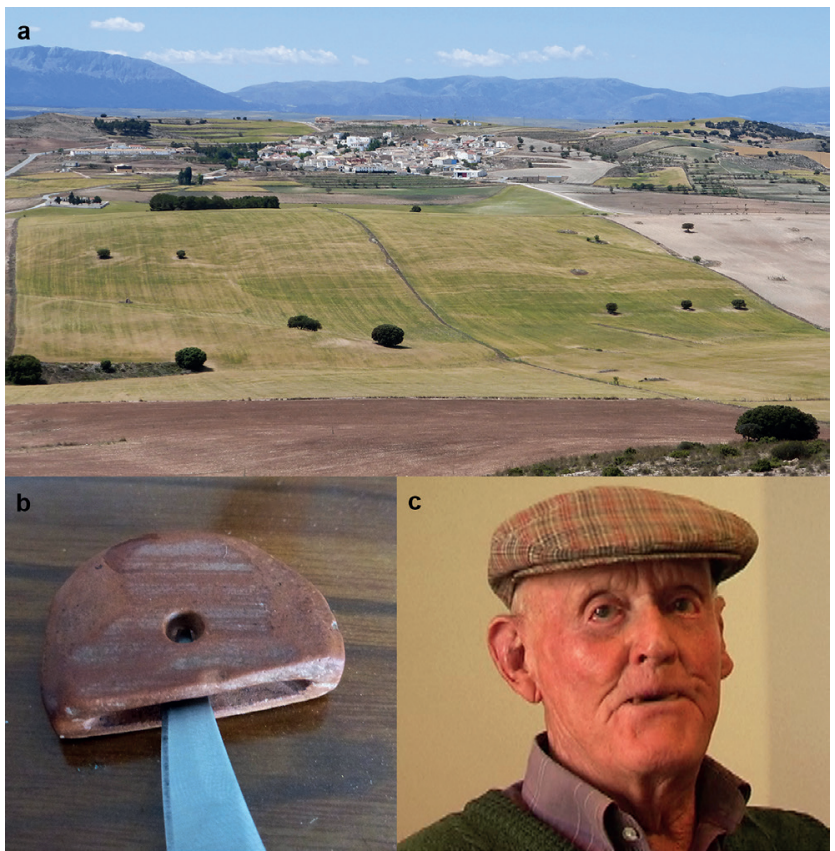

Figura 11. - a. Vista panorámica del pueblo de Topares en Andalucía; b. El silbato de barro llamado pito o boca; c. Un pastor jubilado mostrando como el usa el pito.

Fotos: cortesía de Julien Meyer y Laure Dentel.

(C) Julien Meyer y Laure Dentel. Todos los derechos reservados.

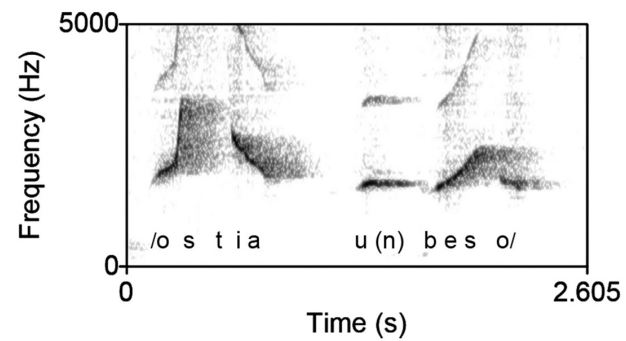

Figura 12. - Palabras «ostia» $y$ «un beso» realizadas por el pastor de la fig. 10 con su pito.

Las realizaciones silbadas de /ostia/ y /un beso/ muestran que las vocales del español silbado en Topares están distribuidas igual a las del Silbo de las diferente Islas Canarias (/i, e, a, o/ en un orden decreciente de frecuencias). Podemos también notar con este ejemplo que /u/ esta silbada relativamente bajo en frecuencias de manera similar al Silbo. André Classe $(1956,1957)$ mencionó que el español de La Gomera y de Andalucía son relativamente similares en pronunciación, con $[\mathrm{u}]$ y $[\mathrm{o}]$ frecuentemente confundidos, considerando que sucede lo mismo en la forma hablada ordinaria. 


\section{Una herencia amenazada}

Un lenguaje silbado proviene de una combinación de factores relacionados tanto con el entorno ecológico como con el entorno social tradicional. Es por eso que sólo sobrevive cuando ambos están bien conservados. Los lenguajes silbados ocupan un lugar emblemático en la diversidad de culturas del mundo. Los hábitats naturales que los albergan, densos bosques y montañas, son hoy esenciales para el planeta, porque allí se concentra la mayor parte de la biodiversidad del mundo. Como práctica lingüística asociada íntimamente a la vida tradicional del campo, hemos identificado que la presencia de un lenguaje silbado en los usos rurales indica que los conocimientos tradicionales asociados al entorno natural están todavía vivos. Por eso, el lenguaje silbado es un indicador de vitalidad de los conocimientos tradicionales sobre algunos de los entornos naturales más importantes y amenazados del planeta. Muchas veces, en los lugares aislados donde hay lenguajes silbados, esos conocimientos tradicionales documentan todavía mejor los ecosistemas locales que las ciencias modernas. Cuando el lenguaje silbado está amenazado significa que las actividades de subsistencia y todos los conocimientos asociados están perdiendo vitalidad. En el Amazonas, por ejemplo, sólo sobrevive en los pueblos en medio del bosque donde todavía se caza, mientras que nadie más aprende a hablar silbando en los pueblos de las zonas deforestadas. En las Islas Canarias ha sobrevivido en las zonas donde había todavía pastores y agricultores de monte, lo que explica que la práctica se perdió más rápidamente en las islas capitalinas, Gran Canaria y Tenerife, que en El Hierro y La Gomera. Hoy en día, los lenguajes silbados se caracterizan por una gran disparidad en las habilidades de quienes los practican. Además, pierden vitalidad en casi todo el planeta debido a los sistemas modernos de comunicación (teléfonos, vehículos motorizados, carreteras) que substituyen su uso. Por eso están en peligro en la mayoría de las zonas que hemos indicado en este artículo.

Consideramos que el silbo es un legado que vale la pena conocer, utilizar, conservar y revalorizar en todos los lugares donde se ha desarrollado en el pasado, conectando y aprovechando los conocimientos de los silbadores tradicionales que lo han practicado localmente, siempre que tengamos la suerte de que esto fuese aún posible. Existen numerosos motivos para su protección, y no solamente por su gran valor histórico y etnográfico, sino por sus múltiples utilidades, tanto presentes como futuras, y por su indudable valor lingüístico y poético. 


\section{REFERENCIAS BIBLIOGRAFICAS}

Abréu Galindo Juan DE, 1977 [1632], Historia de la Conquista de las siete Islas de Canaria, Alejandro Cioranescu (ed.), Santa Cruz de Tenerife, Goya Ed.

Arripe René, 1985, Les siffleurs d'Aas, Pau, Imprimerie de la monnaie.

Bethencourt Alfonso Juan, 1991 [1912], Historia del Pueblo Guanche, tomo 1: Su origen, caracteres etnológicos y lingüísticos, La Laguna, Tenerife, Ed. Francisco Lemus.

Bontier Pierre \& Le Verrier Jean, 1630 [1410-1420], Le Canarien. Histoire de la première descouverte et conqueste des Canaries, faite dès l'an 1402 par Messire Jean de Béthencourt.

Busnel René-Guy, 1964, Documents sur une langue sifflée pyrénéenne, Paris, SFRS.

Busnel René-Guy, 1970, «Recherches expérimentales sur la langue sifflée de Kusköy», Revue de phonétique appliquée, n. $.^{\circ} 14-15$, pp. 41-57.

Busnel René-Guy \& Classe André, 1976, Whistled Languages, Berlin, SpringerVerlag.

Cañadas Asencio Soledad \& Jiménez Morales Inmaculada, 1992, Instrumentos musicales de barro en Andalucía, Granada, Centro de Documentación Musical de Andalucía, Junta de Andalucía.

Chil y Naranjo Gregorio, 1880, Estudios Históricos, climatológicos y patológicos de las Islas Canarias, Paris, Ed. Ernest Leroux.

Classe André, 1956, «Phonetics of the Silbo Gomero», Archivum linguisticum, n. ${ }^{\circ}$, pp. 44-61.

Classe André, 1957, «The Whistled Language of La Gomera», Scientific American, n. ${ }^{\circ} 196$, pp. 111-124.

Coberly Mary Schramm, 1975, «Arbitrary and Natural Features of Non-Tone Language Whistle Speech as Evidence of Diffusion», en F. Ingemann (ed.), Mid America Linguistics Conference Papers Lawrence, University of Kansas, Department of Linguistic, pp. 59-70.

CowAn George, 1976, «Whistled Tepehua», en T. Sebeok y D. J. Umiker-Sebeok (eds), Speech Surrogates: Drum and Whistle Systems, Paris y La Haye, Mouton, pp. 1400-1409.

DíAz Reyes David, 2008, El lenguaje silbado en la Isla de El Hierro, Santa Cruz de Tenerife, Excmo, Cabildo Insular de El Hierro.

DíAz Reyes David, 2011, «El lenguaje silbado en Canarias», Boletín del Archivo Histórico Provincial de Las Palmas, n. ${ }^{\circ}$, Las Palmas de Gran Canaria.

DíAz Reyes David, 2017, El lenguaje silbado en la Isla de El Hierro (segunda revisión ampliada), Tenerife, Le Canarien ediciones, La Orotava.

Espinosa Alonso de (Fray), 1848 [1594], Del origen y milagros de N. S. de Candelaria que aparecio en la isla de Tenerife, con la descripcion de esta isla, Sevilla, Imprenta y Libreria Isleña.

FAnT Gunnar, 1960, Acoustic Theory of Speech Production, The Hague, Mouton \& Co. 
FINkE Peter, 2005, Die Ökologie des Wissens. Exkursionen in eine gefährdete Landschaft, Freiburg y Munich, Alber.

FInKE Peter, 2006, «Die Evolutionäre Kulturökologie: Hintergründe, Prinzipien und Perspektiven einer neuen Theorie der Kultur», Anglia, vol. 124, n. ${ }^{\circ}$, pp. 175-217.

Glas George, 1764, Historia del Descubrimiento y de la Conquista de las Islas Canarias, traducción y notas de P. Nolasco, Santa Cruz de La Palma, 2010.

HASLER Juan, 1960, «El lenguaje silbado», en T. Sebeok y D. J. Umiker-Sebeok (eds), Speech Surrogates: Drum and Whistle Systems, Paris y La Haye, Mouton, pp. 1412-1425.

LAJARD Joseph, 1891, «Le langage sifflé des Canaries», Bulletin de la Société d'anthropologie, vol. 2, n. ${ }^{\circ}$ 1, Paris, pp. 469-483.

Le Clézio Jean-Marie, 1980, Désert, Paris, Gallimard.

López Soler Juan, 1922, De Madrid al Teide, s. n., Madrid.

Lorenzo Perera Manuel J., 2002, El pastoreo en El Hierro. La manada de ovejas, Santa Cruz de Tenerife, Centro de la Cultura Popular Canaria, Excmo, Cabildo Insular de El Hierro.

Marín de Cubas Tomás Arias, 1694, Historia de las siete Islas de Canaria (edición íntegra), La Laguna, Tenerife, Editorial Globo.

MEYER Julien, 2005, Typologie et intelligibilité des langues sifflées : approche linguistique et bioacoustique, Tesis de doctorado, Université Lyon 2, Cyberthèse.

Meyer Julien, 2008, «Acoustic Strategy and Typology of Whistled Languages; Phonetic Comparison and Perceptual Cues of Whistled Vowels», Journal of the International Phonetic Association, vol. 38, n. ${ }^{\circ}$ 1, pp. 69-94.

Meyer Julien, 2012, «La parole sifflée en Amazonie», en A. Anakesa (ed.), Homme, Nature, Patrimonialisation : traditions et pratiques, discours et représentations, connaissances et savoirs dans les cultures plurielles de la Guyane et de la Caraïbe, DVD-ROM, Cayenne, CADEG.

MeYer Julien, 2015, Whistled Language. A Worldwide Inquiry on Human Whistled Speech, Berlin, Springer.

Meyer Julien, Gautheron Bernard \& Ridouane Rachid, 2015, «Whistled Moroccan Tamazight: Phonetics and Phonology», en Proceedings of the 18th International Congress of Phonetic Sciences 2015, Glasgow.

Meyer Julien, Dentel Laure \& Meunier Fanny, 2017, «Categorization of Natural Whistled Vowels by Naïve Listeners of Different Language Background», Frontiers in Psychology, vol. 8, art. 25. <http://dx.doi.org/10.3389/fpsyg.2017. 00025>.

Moore Denny \& Meyer Julien, 2014, «The Study of Tone and Related Phenomena in an Amazonian Language, Gavião of Rondônia», Language Documentation and Conservation, n. ${ }^{\circ}$ 8, pp. 613-636.

Nekitel Otto M., 1992, «A Perceptual Analysis of Abu/-Wam Whistled Speech», en C. Donald, D. T. Laycock, M. Ross \& D. Tryon (eds), The Language Game: Papers in Memory, Cambera, Pacific Linguistics, pp. 299-311. 
Pucheu Gérard, 2015, «Notes sur la parole sifflée en usage dans le Haut-Atlas marocain. Premières observations», Asinag - Revue de l'Institut royal de la culture amazighe, $\mathrm{n} .^{\circ} 10$, pp. 51-66.

QuedenFELDT Max, 1887, «Pfeifsprache auf der Insel Gomera», Zeitschrift für Ethnologie, n. ${ }^{\circ} 19$, pp. 731-741.

Rialland Annie, 2005, «Phonological and Phonetic Aspects of Whistled Languages», Phonology, n. . 22, pp. 237-271.

RiCARD Robert, 1932, «À propos du langage sifflé des Canaries», Hespéris, n. ${ }^{\circ} 15$, pp. 140-142.

RisSET Jean-Claude, 1968, «Sur certains aspects fonctionnels de l'audition», Annales des télécommunications, n. $^{\circ} 23$, pp. 91-120.

SÁnchez González Lidia \& SuÁrez Espino José Pedro (dir.), 1992, Música tradicional y cultura oral en La Aldea de San Nicolás, Gran Canaria, Proyecto Cultural de Desarrollo Comunitario La Aldea, Agrupación Etnográfica La Aldea, Madrid, Tecnosaga.

Schmieder Oscar, 1930, The Settlements of the Tzapotec and Mije Indians, State of Oaxaca, Mexico, Berkeley, University of California Press.

Shadle Christine H., 1983, «Experiments on the Acoustics of Whistling», The Physics Teacher, vol. 21, n. ${ }^{\circ}$ 3, pp. 148-154.

Sicoli Mark A., 2016, «Repair Organization in Chinantec Whistled Speech», Language, vol. 92, n. ${ }^{\circ}$ 2, pp. 411-432. <http://dx.doi.org/10.1353/lan.2016.0028>.

Trapero Maximiano, 1991, «Lenguaje silbado en El Hierro», La Provincia, 7 de noviembre de 1991, p. 38.

Trujillo Carreño Ramón, 2006, El silbo gomero. Nuevo estudio fonológico (edición bilingüe español-inglés), Academia Canaria de la Lengua.

Wilken Gene C., 1979, «Whistle Speech in Tlaxcala (Mexico)», Anthropos, n. ${ }^{\circ}$ 74, pp. 881-888.

\section{Webgrafía}

Perera Mendoza Víctor, 2009: <www.bienmesabe.org/noticia/2009/Julio/vocesdel-saber-juan-marco>.

JimÉnEZ MedinA Antonio et al., 2012: <www.bienmesabe.org/noticia/2012/Abril/ apuntes-etnograficos-del-uso-del-silbo-en-la-isla-de-gran-canaria>.

QuesAdA Jesús, 2017: <www.canarias7.es/siete-islas/gran-canaria/el-lenguaje-delsilbo-pervive-en-la-isla-JF887913>. 